\title{
DOBRAS CUTÂNEAS: LOCALIZAÇÃO E PROCEDIMENTOS
}

\author{
SKIN FOLD \\ LOCALIZATION AND PROCEDIMENTS
}

\author{
AUTORES \\ Alexandre Fernandes Machado' \\ ${ }^{1}$ Laboratório de Fisiologia do Exercício \\ da Universidade Estácio de Sá, \\ Campus Petrópolis - RJ, Brasil \\ DOBRAS CUTÂNEAS: \\ LOCALIZAÇÃO E PROCEDIMENTOS \\ 4(2): $41-45$

\section{PALAVRAS-CHAVE} \\ composição corporal; dobra \\ cutânea; localização; procedimento.
}

\section{KEYWORDS}

body composition; skin fold; localization, procediments.

\section{RESUMO}

O método das dobras cutâneas, conhecido também como método indireto, utiliza-se de equações de regressão para a predição da gordura corporal, onde basea-se na relação entre gordura subcutânea, gordura interna e densidade corporal. Entretanto a sua utilização requer um treinamento prolongado dos avaliadores, para que se obtenha resultados confiáveis. Uma importante limitação na utilização do método de dobras cutâneas e a dificuldade da padronização dos avaliadores em relação aos pontos de medidas e dos procedimentos adotados para a realização da medida. Com isso o objetivo do presente estudo foi realizar uma revisão sobre os pontos de localização para as mensurações das medidas de dobras cutâneas e também quanto aos procedimentos adotados durante o processo de avaliação para minimização dos erros. Inicialmente abordamos a confiabilidade do método, e seqüencialmente os equipamentos para a realização da medida, os procedimentos e a localização das medidas de dobras cutâneas. Concluíndo-se que um dos requisitos para um bom avaliador de dobras cutâneas é o conhecimento dos pontos de reparos e o conhecimento dos procedimentos adotados para o pinçamento de cada dobra cutânea, que são de fundamental importância para o sucesso da medida.

\section{ABSTRACT}

The skin fold method, also known as indirect method, utilized regression equations to predict body fat, where it is based on the relation between subcutaneous fat, internal fat and body density. However its use requires a long period of training from the appraisers, so that the results can be trusted. An important limitation on this method utilization is the difficult in standardizing measure points by the appraisers and the proceedings adopted during the process of evaluation. According to this, this study's objective was to executed a review about the localization points to the skin fold mensuration and also about the adopted proceedings during the evaluation process to minimize the errors. Initially we studied the method trusty and after that the mensuration equipments, the proceedings and the skin fold mensuration localizations. Concluding that one of the requisites to be a good appraiser of skin fold is the knowledge of the repair points and proceedings adopted to get each skin fold are also too important to the measure success. 


\section{INTRODUĈ̣̃O}

Para a análise da composição corporal pode-se fracionar o corpo humano em dois, três ou quatro componentes. Sendo o fracionamento de dois componentes, massa corporal magra (MCM) e massa gorda (MG), o mais utilizado pelo meio científico'.

Para o fracionamento corporal podem ser empregados métodos diretos (análise química de cadáveres), indiretos (hidrometria, espectrometria, densitometria, impedância biolétrica, ressonância nuclear magnética, ultra-sonografia, interactância infravermelho, condutividade elétrica corporal, análise de ativação de nêutrons, análise da absorção de photons], e aqueles conhecidos como duplamente indireto (perimetria e dobras cutâneas].

O método das dobras cutâneas tem sido bastante utilizado no estudo da composição corporal, com objetivo de predizer a gordura corporal relativa (GCR) e a MG, através de equações de regressão ${ }^{2}$. Este método está baseado na relação entre gordura subcutânea, gordura interna e densidade corporal ${ }^{3}$. A utilização do método de dobras cutâneas apresenta outras vantagens, além de não ser um método invasivo, ele tem um baixo custo operacional, aplicabilidade em grandes grupos, rapidez e facilidade na aquisição das medidas ${ }^{1}$. Sendo utilizado amplamente em estudos epidemiológicos ${ }^{4}$.

Porém, sua realização requer treinamento prolongado para que se obtenham resultados confiáveis ${ }^{2}$. Uma importante limitação na utilização do método de dobras cutâneas é a dificuldade de padronização dos avaliadores em relação aos pontos de medidas, e dos procedimentos adotados para a realização da medida, uma vez que o equipamento parece determinar um percentual muito pequeno na variação da medida. Com isso o objetivo do presente estudo foi realizar uma revisão sobre os pontos de localização para as mensuracõos das medidas de dobras cutâneas e também quanto aos procedimentos adotados durante o processo de avaliação para minimização do erro da medida.

\section{Confiabilidade do método}

Utilizando-se de cadáveres, foi observado um coeficiente de correlação igual a 0,84 entre leituras por compassos e a espessura do tecido subcutâneo medido diretamente através de uma incisão realizada no mesmo lugar onde se colocou o compasso 5 . 0 que comprova a elevada efetividade desta técnica. Posteriormente foi desenvolvido um estudo parecido, confirmando a acurácia do método ${ }^{6}$.

Conseqüentemente devemos ter o conhecimento que os valores obtidos apesar de serem bastante válidos, apresentam interferências pela participação de outros tecidos, resultando portanto apenas em valores aproximados e não na quantidade real de gordura subcutânea. Devemos levar em consideração também o modo de como a dobra é destacada e o tipo de compasso utilizado.

Nos indivíduos muito musculosos e obesos, a dobra tem uma característica triangular, com os lados não paralelos na base da dobra, em função da gordura subcutânea não ser facilmente separada do músculo ${ }^{7}$. Com isso aumentando a margem de erro da medida.

A confiabilidade das medidas de dobras cutâneas são afetadas pela: habilidade do avaliador, tipo do compasso utilizado, diferença na quantidade de gordura localizada no tecido adiposo, diferença na espessura da pele, compressibilidade do tecido adiposo, destacamento da dobra e nível de hidratação ${ }^{8}$. Elas não devem ser medidas imediatamente após o exercício, pois, um acúmulo de água extracelular como vasodilatação periférica, certamente aumentaria a espessura da dobra cutânea.

Um outro problema quanto à medida de dobra cutânea é o que se refere à experiência com a própria técnica que os avaliadores devem apresentar. Devemos levar em consideração a influência dos erros intra-avaliadores e interavaliadores. Onde os erros intra-avaliadores são definidos como erros do avaliador em relação a uma medida feita num mesmo indivíduo por ele mesmo, e os erros interavaliadores são as diferenças observadas numa série de medidas realizadas num mesmo grupo de indivíduos por dois ou mais avaliadores.

Aproximadamente de 3 a $9 \%$ da variabilidade em medidas de dobras cutâneas podem estar atribuídas aos erros de medida interavaliadores $^{9}$. 0 tamanho dos erros interavaliadores depende da dobra que se está medindo ${ }^{8}$. Os maiores erros interavaliadores para as dobras cutâneas são: abdome $[8,8 \%]$, da coxa $[7,1 \%$ ) comparados aos do tríceps [3,0\%], subescapular [3,0 a $4,0 \%$ e supra-ilíaca $[4,0 \%]^{9}$.

Para se obter uma boa técnica nas medidas de dobras cutânea devese praticar aproximadamente em 50 a 100 pessoas, para desenvolver um alto nível de habilidade da técnica ${ }^{7}$. Os erros na predição de até $3,5 \%\left[0,0080 \mathrm{~g} / \mathrm{cm}^{3}\right]$ para equações de dobras cutâneas são aceitáveis ${ }^{8}$. 


\section{Equipamentos para a realização da medida}

Para a realização das medidas de dobras cutâneas é necessário um equipamento denominado de compasso de dobras cutâneas. No mercado existem vários tipos e modelos $^{2}$, sendo que somente poucos têm uma aceitação no meio científico internacional, dentre eles - Harpenden (inglês] e o Lange (norte-americano), em função de sua precisão e alta confiabilidade nas medidas ${ }^{10}$.

No Brasil os compassos de dobras cutâneas mais utilizados são o Cescorf (fabricação nacional), que é similar ao Harpenden e o Lange. Eles exercem uma pressão constante de $10 \mathrm{~g} / \mathrm{mm}^{2}$ durante toda a sua escala de medida que vai de 0 a $60 \mathrm{~mm}$. Uma pressão excessiva causa desconforto no avaliado, ou seja, uma sensação de beliscão, além de reduzir significativamente a medida de dobra cutânea.

0 compasso Harpenden requer três vezes mais força para abrir suas hastes. Logo, concluí-se que o tecido adiposo seja mais fortemente comprimido, caracterizando uma medida menor com este tipo de compasso ${ }^{8}$. Essa diferença traduz uma subestimação aproximadamente de $1,5 \%$ na gordura corporal relativa para homens e mulheres ${ }^{11}$.

Posteriormente em outro estudo foram comparados os resultados obtidos por dois diferentes compassos de dobra cutânea (Cescorf, Lange), apresentando um erro padrão de estimativa de $3,3 \%$ $\left[0,0075 \mathrm{~g} / \mathrm{cm}^{3}\right]$, para a densidade corporal. Aplicadas a quatro diferentes equações de predição, propostas originalmente a partir do compasso de Lange. Observou-se que os resultados foram subestimados em $5,2 \%$ a $6,5 \%$ pelo compasso Cescorf ${ }^{12}$.
Recentemente, foi comparado os resultados obtidos por três diferentes compassos de dobras cutâneas [Cescorf, Lange, Sanny), sendo o compasso Sanny de fabricação nacional, assim como o Cescorf. Onde os pesquisadores observaram diferenças significativas quando comparado os resultados obtidos pelo Lange com os outros dois tipos de compassos (Cescorf, Sanny), embora os resultados encontrados entre os compassos Cescorf e Sanny não apresentassem diferenças significativas nos resultados ${ }^{2}$.

\section{Procedimentos para a realização da medida de dobra cutânea}

As altas correlações com os métodos indiretos e seu baixo custo operacional foram os principais motivos da grande utilização deste método. Porém a aplicação deste método de maneira eficiente requer que o avaliador siga alguns procedimentos com o objetivo minimizar os erros de medida, sendo eles:

Todas as medidas devem ser realizadas no lado direito do corpo; identificar e marcar com uma caneta ou lápis apropriado o local da medida; o pinçamento da dobra cutânea deve ser feito com a mão esquerda e com os dedos polegar e indicador a $\pm 1 \mathrm{~cm}$ acima do local marcado da medida; colocar as hastes do compasso perpendicular à dobra, $\pm 1 \mathrm{~cm}$ abaixo do local pinçado, e soltar lentamente as hastes do compasso; as hastes do compasso não deveram passa do ponto pinçado pelos dedos; manter a dobra pressionada enquanto à medida é realizada; fazer à leitura no compasso \pm 3 segundos após a pressão ter sido aplicada na dobra; afastar as hastes do compasso para remove-lo e fecha-las lentamente; ler o mostrador em sua escala mais próxima; realizar no mínimo duas medidas para cada local, caso os valores diferenciem em mais de $10 \%$, realizar novas medidas; realizar as medidas em uma ordem rotativa, em vez de leituras consecutivas; realizar as medidas sob a pele seca e sem loções; não medir as dobras imediatamente após o exercício; evitar usar compassos de plástico caso se trate de um avaliador inexperiente; treinar com outros avaliadores e comparar seus resultados ${ }^{8}$.

\section{Localização da medida de dobra cutânea}

As medidas devem ser realizadas em várias regiões para se obter um termo médio da sua gordura corporal total. A distribuição dos pontos de dobras cutâneas pelo corpo se justifica pelo fato da distribuição do tecido adiposo subcutâneo não apresentar uniformemente em termos de quantidade por todo o corpo, existindo a necessidade do emprego de medidas realizadas em diferentes locais para se obter uma visão mais significativa do tecido gorduroso ${ }^{8}$.

A exata localização de cada ponto de medida de dobra cutânea é extremamente importante, pois, a sua distribuição o leva a índices que pode atingir erros até duas vezes mais do que os erros intra-avaliadores. Alguns pesquisadores analisaram que com o deslocamento proximal, distal, lateral e medial de 2,5 cm da dobra de tríceps, poderia acarretar uma alteração na leitura aproximadamente de 2 a $3 \mathrm{~mm}$, o que é bastante significativo ${ }^{13}$. Tornando-se de fundamental importância para o avaliador o conhecimento em anatomia.

Abaixo (tabela 1) estão descritos detalhadamente as dobras cutâneas, seus locais de medida e seus respectivos procedimentos. 


\begin{tabular}{lcc}
\hline $\begin{array}{l}\text { Dobra } \\
\text { Cutãnea }\end{array}$ & $\begin{array}{c}\text { Sentido } \\
\text { da Dobra }\end{array}$ & $\begin{array}{c}\text { Referência } \\
\text { Anatômica }\end{array}$ \\
\hline Subescapular & $\begin{array}{c}\text { Diagonal } \\
\text { ou } \\
\text { Obliquo }\end{array}$ & * Ângulo inferior da escápula \\
& & * * Borda medial da escápula \\
& e ângulo inferior da escápula
\end{tabular}

Procedimento

\begin{tabular}{|c|c|c|c|}
\hline Tríceps & $\begin{array}{c}\text { Vertical } \\
\text { (linha média) }\end{array}$ & Ponto Meso-umeral & Dobra na região posterior do braço $0^{7,14,15,16}$. \\
\hline Bíceps & $\begin{array}{c}\text { Vertical } \\
\text { (linha média) }\end{array}$ & Ponto Meso-umeral & Dobra na região anterior do braço ${ }^{7,14,15,16}$. \\
\hline Peitoral & Diagonal & Linha axilar anterior e mamilo & $\begin{array}{l}\text { * Dobra localizada no ponto médio entre a linha axilar } \\
\text { anterior e o mamilo } p / \text { ambos os sexos }{ }^{14} \text {. } \\
\text { * * Dobra localizada no ponto médio entre a linha axilar } \\
\text { anterior e o mamilo } p / \text { sexo masculino, e } 1 / 3 \mathrm{p} / \text { o sexo } \\
\text { feminino } 0^{7,16} \text {. }\end{array}$ \\
\hline Axilar média & $\begin{array}{l}\text { *Horizontal } \\
\text { ou } \\
* * \text { Vertical } \\
\text { ou } \\
* * * \text { Obliqua }\end{array}$ & $\begin{array}{l}\text { Junção xifoesternal } \\
\text { e linha axilar média }\end{array}$ & $\begin{array}{l}\text { *Dobra localizada na linha axilar média e ao nível da } \\
\text { junção xifoesternal }{ }^{14} \text {. } \\
\text { * * Dobra localizada em cima da linha axilar média ao } \\
\text { nível do processo xifóide }{ }^{7,16} \text {. } \\
\text { *** Dobra localizada no ponto de intersecção da linha } \\
\text { axilar média com uma linha imaginária que passa pelo } \\
\text { apêndice xifóide }{ }^{15} \text {. }\end{array}$ \\
\hline Supra-ilíaca & $\begin{array}{l}\text { * Obliqua } \\
\text { ou } \\
\text { * * Diagonal }\end{array}$ & Crista ilíaca & $\begin{array}{l}\text { * Dobra localizada em cima da linha axilar média e logo } \\
\text { acima da crista ilíaca }{ }^{14} \text {. } \\
\text { * * Dobra localizada logo acima da crista ilíaca em um } \\
\text { ponto coincidente com a linha axilar anterior }{ }^{7,15,16} \text {. }\end{array}$ \\
\hline Abdome & $\begin{array}{l}\text { *Horizontal } \\
\text { ou } \\
* * \text { Vertical }\end{array}$ & Cicatriz umbilical & $\begin{array}{l}\text { * Dobra lateralmente a } 3 \mathrm{~cm} \text { de distância da cicatriz } \\
\text { umbilical e } 1 \mathrm{~cm} \text { abaixo do centro da cicatriz umbilical }{ }^{14} \text {. } \\
\text { * } \text { * Dobra localizada lateralmente a } 2 \mathrm{~cm} \text { da cicatriz } \\
\text { umbilical }{ }^{7,15} \text {. } \\
\text { * * Dobra localizada lateralmente de } 3 \text { a } 5 \mathrm{~cm} \text { da cica- } \\
\text { triz umbilical }{ }^{16} \text {. }\end{array}$ \\
\hline Coxa & $\begin{array}{l}\text { Vertical (linha } \\
\text { média femoral) }\end{array}$ & $\begin{array}{c}\text { * Dobra inguinal } \\
\text { e } \\
\text { * * borda superior da patela }\end{array}$ & $\begin{array}{l}\text { * Dobra localizada na região anterior da coxa no ponto } \\
\text { médio femoral }{ }^{7,14,16} \text {. } \\
\text { * * Dobra localizada na região anterior da coxa a } 1 / 3 \\
\text { da prega inguinal e borda superior da patela }{ }^{15} \text {. }\end{array}$ \\
\hline $\begin{array}{l}\text { Panturrilha } \\
\text { medial }\end{array}$ & $\begin{array}{l}\text { Vertical (região } \\
\text { média da perna) }\end{array}$ & $\begin{array}{l}\text { Máxima circunferência } \\
\text { da perna }\end{array}$ & $\begin{array}{l}\text { Dobra localizada no ponto de maior circunferência da } \\
\text { perna na parte medial da mesma. } 0 \text { avaliado deverá } \\
\text { estar sentado }{ }^{7,14,15,16} \text {. }\end{array}$ \\
\hline
\end{tabular}




\section{CONCLUSÃO}

As medidas de dobra cutânea são amplamente utilizadas para predição da gordura corporal, em função de seu baixo custo operacional, porém sua técnica requer um alto treinamento por parte dos avaliadores para minimização dos erros. 0 conhecimento dos pontos de reparos e procedimentos adotados para o pinçamento de cada dobra cutânea também são de fundamental importância para o sucesso da técnica.

Recomenda-se, para minimização dos erros, além de um treinamento periódico sobre os avaliadores, que os pontos de reparos, e os procedimentos adotados para o pinçamento de cada dobra sejam compatíveis com os protocolos escolhidos para determinação da gordura corporal, assim como o compasso de dobras cutâneas escolhido para a mensuração das medidas.

\section{CORRESPONDÊNCIA}

Alexandre Fernandes Machado

Rodovia Raposos Tavares, número 3175, Bloco G, Ap. 13, Jardim Pinheiros, São Paulo, SP - Brazil

CEP. 05577-100

E-mail: xdmachado@gmail.com

\section{REFERÊNCIAS}

1. Carvalho ABR, Pires Neto CS (1999). Composição corporal através dos métodos de pesagem hidrostática e impedância bioelétrica em universitários. Revista Brasileira de Cineantropometria \& Desempenho Humano. 1(1): 18-23.

2. Barillo JLM, Burger M, Machado AF [2005). Análise da gordura corporal obtida por diferentes tipos de compassos de dobras cutâneas. Revista Meta Science. 2(3): 38-40.

3. Petroski EL (1995). Desenvolvimento e validação de equações generalizadas para a predição da densidade corporal. Tese de Doutorado. UFSM-RS, Universidade Federal de Santa Maria.

4. Sichieri R, Fonseca VM, Lopes CS (1999). Como medir a confiabilidade de dobras cutâneas. Revista Brasileira de Epidemiologia. 2(1): : 82-89.

5. Lee MMC, Ng CK (1965). Postmoetren studies skinfold capiler measurent and actual thickness of skimand subcutaneous tissue. Human Biology.

6. Martin AD (1985). Predietion of body fat by skinfold caliper: assumptions and cadaver evidence. Inter. Journal of medicine.

7. Jackson AS, Pollock ML (1985). Pratical assessment of body composition. The Physician and sport medicine. 13: 256-262.

8. Heyward VH, Stolarczyk LM (2000). Avaliação da composição corporal. Manole, São Paulo.
9. Lohmam TG, Pollock ML (1984). Methodological factors and the prediction Of body fat in female athletes. Medicine and science in sport and exercise. 16.

10. Whitehead JR (1990). A study of measurement variation among different skinfold calipers. Br J Phys Educ. 7: 10-14.

11. Gruber JJ, Pollock ML (1990). Coparison of harpenden and lange calipers In predicting body composition research. Quarterly for exercise and sport. 61: 456-461.

12. Cyrino ES, Okano AH, Glaner MF, Romanzini M, Gobbo LA, Makoski A, Bruna N, Melo JC, Tassi GN (2003). Impacto da utilização de diferentes compassos de dobras cutâneas para análise da composição corporal. Revista Bras Med Esporte. 9(3): 145-149.

13. Ruiz L, Colley JRT, Hamilton PJS (1971). Measurent of tríceps skinfold thickness, investigation of souces of variation. British journal of preventive and social medicine.

14. Lohman TG, Roche AF, Martorell R (1991). Anthropometric Standardization refence manual. Abridged editon Human Kinetics books. Ilinois. 15. Guedes DP (1985). Tentativa de validação de equações para predição dos valores de densidade corporal com base nas espessuras de dobras cutâneas em universitários. Revista Brasileira de Ciência do Esporte. 6: 182-191.

16. Ross WD, Carr RV, Carter JEL (2000). Antropometry illustrated: a browser ased interactive textbook and learning system. The human animal series, v1. 